\title{
MEASURE REQUIREMENTS ON DISTRIBUTIVE LATTICES FOR BOOLEAN ALGEBRAS AND TOPOLOGICAL APPLICATIONS
}

\author{
CARLOS C. HUERTA \\ (Communicated by James E. West) \\ Dedicated to "Doc" and Ma.
}

\begin{abstract}
In this note we give a measure requirement for a distributive lattice to be complemented, and therefore a boolean algebra. Applying this result and using measure style arguments, we get various topological results.
\end{abstract}

Given a distributive lattice containing 0,1 , we wish to give two different but connected requirements for the lattice to be complemented and therefore a boolean algebra. What makes this note of particular interest is that our main result is given in terms of measures. It is the measure representation which has been shown to be of great value in investigating the topological properties of the lattice.

We first define an anti-isomorphism $T: \mathscr{L}_{1} \rightarrow \mathscr{L}_{2}$ where $\mathscr{L}_{1}, \mathscr{L}_{2}$ are distributive lattices containing 0,1 . We require $T$ to be one-to-one and onto such that

(a) $T(a \cup b)=T(a) \cap T(b)$ and

(b) $T(a \cap b)=T(a) \cup T(b)$ for all $a, b \in \mathscr{L}_{1}$.

We now take $F$, an arbitrary collection of elements from $\mathscr{L}_{1}$ and, considering the set $C F=\left\{a \in \mathscr{L}_{1} \mid a \notin F\right\}$, we can show two facts, generalized from Bourbaki [1]:

Fact 1. Let $T: \mathscr{L}_{1} \rightarrow \mathscr{L}_{2}$ be an anti-isomorphism; then $T[C F]$ is a prime $\mathscr{L}_{2}$-filter iff $F$ is a prime $\mathscr{L}_{1}$-filter.

Fact 2. Let $T: \mathscr{L}_{1} \rightarrow \mathscr{L}_{2}$ be an anti-isomorphism. If we take a $a \in \mathscr{L}_{1}$ such that $a \neq 0$, then $H=\left\{T(b) \mid T(a) \cup T(b)=1, b \in \mathscr{L}_{1}\right\}$ is an $\mathscr{L}_{2}$-filter.

Now, assuming all prime $\mathscr{L}_{1}$-filters are maximal, by Fact 2 we can create $H$, an $\mathscr{L}_{2}$-filter; extending $H$ to $G$, an $\mathscr{L}_{2}$-ultrafilter, and considering $T^{-1}$ [CG], we realize that if $\mathscr{L}_{1}$ is not complemented we violate Fact 1 . With this, we can obtain a theorem which also follows from Bourbaki [1].

Received by the editors November 12, 1987 and, in revised form, April 19, 1988.

1980 Mathematics Subject Classification (1985 Revision). Primary 06D10, 06D99; Secondary 28A60, $28 \mathrm{C} 15$.

Key words and phrases. Anti-isomorphism, filter, zero-one valued measures.

(C) 1989 American Mathematical Society $0002-9939 / 89 \$ 1.00+\$ .25$ per page 
Theorem 1. Let $\mathscr{L}$ be a distributive lattice containing 0,1 and suppose all prime $\mathscr{L}$-filters are maximal; then $\mathscr{L}$ is a boolean algebra.

We denote $I(\mathscr{L})$ as the collection of all zero-one valued measures defined on the boolean algebra $\mathscr{A}(\mathscr{L})$ generated by the lattice $\mathscr{L}$. We denote $\operatorname{IR}(\mathscr{L}) \subseteq$ $I(\mathscr{L})$ as the set of all zero-one valued measures defined on $\mathscr{A}(\mathscr{L})$ where $\mu \in I R(\mathscr{L})$ if

$$
\mu(A)=\inf _{\substack{L^{\prime} \supseteq A \\ L \in \mathscr{L}}} \mu\left(L^{\prime}\right)=\sup _{\substack{L \subseteq A \\ L \in \mathscr{L}}} \mu(L)
$$

for all $A \in \mathscr{A}(\mathscr{L})$. Now, using Theorem 1 and a result from Bachman and Cohen [2], we get the result:

Theorem 2. Let $\mathscr{L}$ be a distributive lattice containing 0,1 and suppose $I(\mathscr{L})=$ $\operatorname{IR}(\mathscr{L})$; then $\mathscr{L}$ is a complemented lattice.

We now can get some immediate topological results from Theorem 2.

(1) If we take a topological space $X$ and let $\mathscr{L}=0$, the lattice of open sets, then if $I(\mathscr{L})=I R(\mathscr{L}), X$ is extremely disconnected.

(2) If we consider $V(L)=\{\mu \in I(\mathscr{L}) \mid \mu \in(L)=1\}$ and form $V(\mathscr{L})=$ $\{V(L) \mid L \in \mathscr{L}\}$, we can topologize $I(\mathscr{L})$ by taking arbitrary intersections of $V(\mathscr{L})$; see [3]. It is known [4] that the topological space $(I(\mathscr{L}), \tau V(\mathscr{L}))$ is $T_{0}$. It follows from the above that if $I(\mathscr{L})=I R(\mathscr{L})$ then $(I(\mathscr{L}), \tau V(\mathscr{L}))$ is $T_{2}$.

We call the lattice $\mathscr{L}$ cocomplement generated if for all $L \in \mathscr{L}$ we can write $L=\bigcup_{n} L_{n}^{\prime}, L_{n} \in \mathscr{L}$. Again, using Theorem 2 and solely measure arguments we have

Theorem 3. Let the lattice $\mathscr{L}$ be countably compact and cocomplement generated; then $\mathscr{L}$ is complemented, and therefore a boolean algebra.

We again note that it is this measure approach to lattice properties and lattice topologies that is of interest. Further applications will be published elsewhere.

\section{REFERENCES}

1. N. Bourbaki, Elements of mathematics, general topology, part I, Hermann, Paris, 1966, pp. 130-132.

2. G. Bachman and R. Cohen, Regular lattice measures and repleteness, Comm. Pure Appl. Math., 26 (1973), 587-599.

3. R. Cohen, Lattice measures and topologies, Ann. Mat. Pura Appl. IV, 109 (1976), 147-164.

4. G. Bachman and P. D. Stratigos, On general lattice repleteness and completeness, Illinois J. Math., 27 (1983), 535-561.

Jerusalem School of Management, Mishkalov 8/12, Harnof, Jerusalem, 95402 Israel 